\section{artigo original}

\author{
João R.I. Carneivo \\ Maria C. Kushnir \\ Eliete L.S. Clemente \\ Marcelo G. Brandão \\ Marilia de B. Gomes
}

Disciplina de Diabetes e Metabologia e Unidade Clínica de Adolescentes (MCK), Universidade do Estado do Rio de Janeiro (UERJ), Rio de Janeiro, $R J$.

Recebido em 29/10/99

Revisado em 10/03/00 e 26/05/00

Aceito em $02 / 06 / 00$

\title{
Obesidade na Adolescência: Fator de Risco para Complicações Clinico-Metabólicas
}

\section{RESUMO}

Sessenta e seis adolescentes, sendo 38 obesos (25F/13M) e 28 não obesos (23F/5M), pareados pelo sexo, idade, cor; nivel de escolaridade e estágio de maturação sexual, foram submetidos a um inquérito clínicodemográfico e à avaliação clínico-laboratorial. A relação cinturaquadril foi maior no grupo dos obesos do que nos não obesos $(0,86 \pm 0,08$ vs. 0,74 $\pm 0,04 ; p<0,01$ ), assim como as medidas de pressão arterial sistóli-

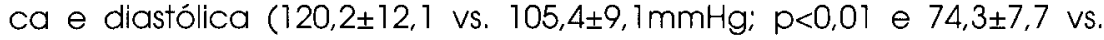
$65,5 \pm 9,4 \mathrm{mmHg} ; \mathrm{p}<0,01$, respectivamente). A lesão dermatológica acanthosis nigricans predominou no grupo dos obesos $(n=24,63,2 \%)$. Os obesos apresentaram valores de HDL-colesterol inferiores aos dos não

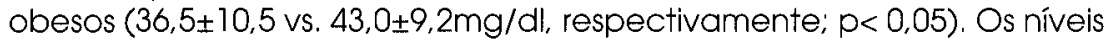
de triglicerídeos e ácido úrico foram maiores no grupo dos obesos se comparado aos não obesos (124,6 480,0 vs. $74,2 \pm 31,4 \mathrm{mg} / \mathrm{dl}$, respectiva-

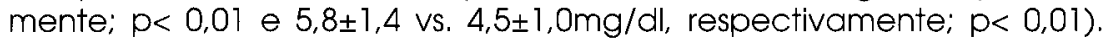
Não houve diferença nos valores de colesterol, LDL colesterol e na glicemia basal entre os dois grupos. Os adolescentes obesos procuraram tratamento na maioria das vezes movidos por uma preocupação com a saúde $(n=15,39,5 \%)$. Um número significativo desses adolescentes já havia feito uso de medicamentos visando a perda ponderal no passado. Concluímos que a obesidade na adolescência pode estar relacionada a um perfil clínico-metabólico desfavorável, caracterizado por níveis mais elevados de pressão arterial sistólica e diastólica, triglicerídeos, ácido úrico, e mais reduzidos de HDL-colesterol; e ainda pela presença de acanthosis nigricans. (Arq Bras Endocrinol Metab 2000;44/5: 390-396)

Unitermos: Obesidade; Adolescente; Colesterol; Triglicérides; Ácido úrico; Pressão arterial

\begin{abstract}
Sixty six adolescents, 36 obese (25F/13M) and 28 non-obese (23F/5M), matched for sex, age, race, education level and Tanner stage of sexual maturation were submitted to a demographical inquire, clinical evaluation and laboratory analysis. The waist-to-hip ratio $(0.86 \pm 0.08$ vs. $0.74 \pm 0.04 ; p<0.01)$ and systolic and diastolic blood pressures $(120.2 \pm 12.1$ vs. $105.4 \pm 9.1 \mathrm{mmHg} ; p<0.01$ and $74.3 \pm 7.7$ vs. $65.5 \pm 9.4 \mathrm{mmHg} ; p<0.01)$, were higher in the obese group as compared to the non-obese group. Acanthosis nigricans predominated in the obese group ( $n=24 ; 63.5 \%$ ). Levels of HDL-cholesterol were lower in obese adolescents $(36.5 \pm 10.5 \mathrm{vs}$. $43.0 \pm 9.2 \mathrm{mg} / \mathrm{dl} ; \mathrm{p}<0.05$ ), whereas levels of triglycerides and uric acid were higher (124.6 \pm 80.0 vs. $74.2 \pm 31.4 \mathrm{mg} / \mathrm{dl} ; \quad p<0.01$ and $5.8 \pm 1.4$ vs, $4.5 \pm 1.0 \mathrm{mg} / \mathrm{dl} ; \mathrm{p}<0.01$ ), respectively. No differences were found in cholesterol, LDL-cholesterol and basal glucose between the two groups. Obese adolescents sought for treatment because of concern about their health $(n=15,39.5 \%)$. Most of them had already used antiobesity drugs in the past. We conclude that obesity in adolescence seems to be associated with a clinical and metabolic profile of insulin resistance with
\end{abstract}


acanthosis nigricans and higher levels of systolic and diastolic blood pressure, trygliceride and uric acid as well as low levels of HDL-cholesterol. (Arq Bras Endocrinol Metab 2000;44/5: 390-396)

Keywords: Obesity; Adolescent; Cholesterol; Tryglicerides; Uric acid; Blood pressure

A OBESIDADE VEM SENDO CONSIDERADA um grande problema de saúde pública das sociedades desenvolvidas e em desenvolvimento do mundo contemporâneo. Nos Estados Unidos da América (EUA), mais de $40 \%$ da população adulta encontra-se acima do peso (1). Estudos epidemiológicos apontam para um importante crescimento ponderal da população americana nas duas últimas décadas $(1,2)$. Naquele país, um número bastante significativo de pessoas estão acima da faixa de peso ideal e encontram-se à mercê de diversas complicações clínico-metabólicas. Estes dados também refletem a realidade de outros países desenvolvidos. A obesidade é hoje motivo de preocupação para as autoridades de saúde britânicas. Sua prevalência praticamente dobrou de 1980 a 1991 (3).

No Brasil, observou-se um importante crescimento ponderal no período compreendido entre 1974 e 1989, estimando-se hoje que um terço da população brasileira encontra-se acima da faixa de peso ideal (4). A melhoria das condições de vida, em especial o maior acesso à alimentação por camadas mais pobres da população e a diminuição do gasto diário de energia proporcionado por avanços tecnológicos vêm sendo apontados como responsáveis pelo incremento na prevalência da obesidade na população brasileira (4).

A adolescência corresponde a um período da vida no qual ocorrem profundas modificações no crescimento e maturação do ser humano. Neste período de transição, que segundo a WHO corresponde ao

Tabela 1. Características demográficas das populações estudadas.

\begin{tabular}{lcc}
\hline Variável & $\begin{array}{c}\text { Obesos } \\
(\mathbf{n = 3 8 )}\end{array}$ & $\begin{array}{c}\text { Não obesos } \\
(\mathbf{n}=\mathbf{2 8})\end{array}$ \\
\hline $\begin{array}{l}\text { Feminino/masculino } \\
\text { Branco/não branco }\end{array}$ & $25 / 13$ & $23 / 5$ \\
$\begin{array}{l}\text { ldade (anos) } \\
\begin{array}{l}\text { Escolaridade } \\
\text { (c/d/e/f) }\end{array}\end{array}$ & $14,6 \pm 1,8$ & $17 / 11$ \\
$\begin{array}{l}\text { Tanner } \\
\mathbf{2 / 3 / 4 / 5}\end{array}$ & $4 / 21 / 13 / 0$ & $3 / 16 / 8 / 1$ \\
\hline
\end{tabular}

Valores expressos em média \pm SD; Tanner, estágio de maturação sexual segundo Tanner. IMC. Índice de massa corporal. Escolaridade: c, $1^{\circ}$ ciclo do $7^{\circ}$ grau; d, $2^{\circ}$ ciclo do $1^{\circ}$ grau; $e, 2^{\circ}$ grau em curso; f, $2^{\circ}$ grau completo. intervalo dos 10 aos 19 anos (5), é extremamente difícil estabelecer uma definição precisa para obesidade e excesso ponderal. Durante o processo de crescimento e maturação, as proporções corporais, a massa óssea e a relação entre tecido gorduroso e muscular sofrem variações de diferentes magnitudes e velocidades (6). Por anteceder de imediato a idade adulta, a época da adolescência deve ser considerada de grande importância para que se estabeleçam intervenções que possam modificar riscos futuros (7).

Crianças e adolescentes obesos serão adultos obesos em potencial. Foi demonstrado um risco cumulativo para obesidade na idade adulta que varia de 2,33 para crianças com peso acima do percentil 90 em seus seis primeiros meses de vida até 6,55 , observado em meninos e meninas entre 10 e 13 anos de idade $(8,9)$.

De etiologia multifatorial, a obesidade iniciada antes da idade adulta parece guardar importante relação com fatores hereditários (10), estando o sucesso do tratamento muitas vezes condicionado à participação dos pais no programa terapêutico utilizado (11). Uma vez que adolescentes obesos muito provavelmente permanecerão acima do peso na idade adulta, estarão pois sujeitos ao desenvolvimento das diversas complicações clínico-metabólicas encontradas em adultos obesos (7). Além disso, este grupo de pacientes já apresenta antes mesmo de atingir a maturidade, fatores de risco clínicometabólicos para desenvolvimento da síndrome de resistência insulínica. A obesidade predispõe o adolescente à elevação dos níveis séricos de VLDL, LDL, triglicerídeos (TG), apolipoproteína B e à diminuição de HDL-colesterol e apolipoproteína A-1 (12,13); níveis elevados de pressão arterial sistólica e diastólica (14) e ao desenvolvimento de acanthosis nigricans, lesão dermatológica considerada indicadora de severidade da resistência insulínica (15).

O objetivo deste trabalho foi determinar associações entre a obesidade na adolescência e alterações clínico-metabólicas indicadoras de morbi-mortalidade.

\section{MATERIAL E MÉTODOS}

Foram estudados 66 adolescentes, sendo 38 obesos ( 25 do sexo feminino e 13 do masculino) e 28 não obesos ( 23 do sexo feminino e 5 do masculino), pareados pelo sexo, cor, idade, estágio de maturação sexual e nível de escolaridade. O grupo de obesos foi constituído de pacientes que procuraram o Programa de Atenção e Prevenção da Obesidade (PAPO) na adolescência, do Núcleo de Estudos dos Adolescentes (NESA)/UERJ no período de outubro de 1996 a novembro de 1998. O grupo de não obesos consti- 
tuiu-se de indivíduos do ciclo de relacionamento dos pacientes (parentes e amigos) e estagiários de setores administrativos do HUPE.

Os adolescentes foram submetidos a um questionário padrão abordando hábitos alimentares e de vida, além de informações a respeito dos motivos da procura ao serviço (no caso dos obesos), histórico de tratamentos anteriores, histórico de obesidade em familiares de $1^{0}$ grau e a uma avaliação clínico-laboratorial prévia ao início do tratamento. Os familiares e/ou responsáveis auxiliaram no fornecimento das informações. O grau de escolaridade foi definido como: a, para analfabetos; b, para alfabetizados; c, para o $1^{\circ}$ ciclo do $1^{\circ}$ grau; d, para o $2^{\circ}$ ciclo do $1^{\circ}$ grau; e, para $2^{\circ}$ grau em curso; f, para o $2^{\circ}$ grau completo. A pressão arterial sistêmica foi obtida com o paciente em posição supina no tempo zero e após 5 minutos de repouso, no braço não dominante e sempre pelo mesmo examinador e com o mesmo equipamento. Nas aferições foi utilizado um esfigmomanômetro de coluna de mercúrio padronizado e calibrado e manguitos adequados às dimensões corpóreas. A pressão diastólica foi considerada como o desaparecimento dos sons de Korotkoff (fase 5). O indivíduo foi considerado hipertenso quando a média das aferições da pressão arterial sistólica (PAS) e/ou pressão arterial diastólica (PAD) estava acima do percentil 95 para a idade e sexo (16). A medida do peso foi obtida com o indivíduo sem sapatos e utilizando roupas leves sobre balança Filizola modelo 31 série $3134, \mathrm{n}^{\mathrm{O}} 109835$. A medida da altura foi obtida com o indivíduo sem calçados, sobre um estadiômetro de Harpenden. A prega tricipital foi obtida no ponto médio entre o processo acromial e o olecrânio, na face posterior do membro não dominante (17), sendo considerada a média de 3 medidas. A prega subescapular foi obtida obliquamente ao eixo longitudinal, seguindo a orientação dos arcos costais, no ponto $2 \mathrm{~cm}$ abaixo do ângulo inferior da escápula do lado do membro não dominante (17), sendo considerada a média de 3 medidas. Foi utilizado um paquímetro de Lange para a aferição das pregas. A medida da cintura foi obtida na menor circunferência entre o gradil costal e a cicatriz umbilical. A medida do quadril foi obtida na maior circunferência da extensão posterior da região glútea (17), sendo considerada a média de 3 medidas. Nas aferições foi utilizada fita métrica com precisão de $0,5 \mathrm{~cm}$. A relação cintura-quadril (RCQ) foi obtida através da divisão da circunferência da cintura pela do quadril. $O$ índice de massa corporal (IMC) foi calculado dividindo-se o peso $(\mathrm{Kg})$ pela altura ao quadrado $\left(\mathrm{m}^{2}\right)$, utilizando- se os limites definidos para população brasileira, sendo considerados obesos aqueles com IMC acima do percentil 90 (18). As medidas antropométricas foram avaliadas pelo mesmo examinador. Determinou-se o estágio de maturação sexual através da classificação de Tanner do desenvolvimento puberal. Os dados antropométricos das populações estudadas são apresentados na tabela 2 .

O diagnóstico da lesão dermatológica acanthosis nigricans, caracterizada por papilomatose, hiperceratose e hiperpigmentação da epiderme (19), se deu através de exame clínico, sendo investigadas as seguintes regiões: cervical, axilar, antecubital, face interna da coxa e superfícies extensoras falangeanas. Foram considerados portadores da lesão aqueles que a manifestaram em pelo menos uma das regiões citadas.

A glicemia foi dosada pelo método enzimático colorimétrico automatizado (Aparelho COBAS MIRA da Roche Diagnóstica), sendo os valores de referência no soro ou plasma (em jejum) de 70 a $105 \mathrm{mg} / \mathrm{dl}$. O colesterol total foi dosado pelo método enzimático colorimétrico com colesterol-estearase, colesterol oxidase e reação catalisada pela peroxidase, e os triglicerídeos pelo método enzimático colorimétrico GPO/PAP, sendo os valores limítrofes de referência no soro ou plasma (em jejum) menores que $200 \mathrm{mg} / \mathrm{dl}$ para o colesterol e menores que $130 \mathrm{mg} / \mathrm{dl}$ para os triglicerídeos. O HDL-colesterol foi dosado com kit HDL-Cholesterol Direct FS (Fluid Stable) quantitative determination of High Density Lipoprotein without Centrifugation, da DiaSys ${ }^{\circledR}$. O LDLcolesterol foi calculado pela fórmula de Friedwald (20) $[$ LDL-colesterol $=$ colesterol total - (HDL-colesterol + TG/5)] para valores de TG inferiores a $400 \mathrm{mg} / \mathrm{dl}$. A dosagem de ácido úrico foi obtida por método enzimático colorimétrico da SMT ${ }^{\circledR}$. Os valores de referência são: 2,4 a $5,7 \mathrm{mg} / \mathrm{d}$ l para o sexo feminino e 3,4 a $7,0 \mathrm{mg} / \mathrm{dl}$ para o sexo masculino.

Os dados coletados foram arquivados no programa de epidemiologia EPI INFO, versão 6,02 de outubro de 1992 e analisados pelo programa SPSS for Windows. O teste de Shapiro foi utilizado para se testar a normalidade da distribuição de variáveis contínuas e o de Mann-Whitney foi aplicado para comparação entre duas amostras independentes. Nas demais comparações utilizaram-se os testes $t$ de Student e a análise de variança (ANOVA). Nas tabelas de contingência utilizou-se o teste do Qui-quadrado com correção de Yates ou o teste exato de Fisher. Os dados são apresentados como média \pm desvio padrão. Considerou-se como significante um valor de p bicaudal $<0,05$. 


\section{RESULTADOS}

O grupo de obesos apresentou medida da cintura superior à encontrada no grupo de não obesos, respectivamente $(98,9 \pm 15,8 \mathrm{~cm}$ vs. $66,9 \pm 5,2 \mathrm{~cm}, \mathrm{p}=0,0000)$. A relação cintura-quadril também foi maior no grupo de obesos do que no grupo controle, respectivamente $(0,86 \pm 0,08$ vs. $0,74 \pm 0,04, p=0,0000)$. As medidas de pressão arterial sistólica após 5 minutos de repouso (PAS 5') foram maiores no grupo de obesos quando comparados ao grupo de não obesos, respectivamente $(120,2 \pm 12,1 \mathrm{mmHg}$ vs. $105,4 \pm 9,1 \mathrm{mmHg}, \mathrm{p}=0,0000)$. Observamos pressão arterial diastólica após 5 minutos de repouso (PAD 5') superior em obesos quando comparados aos não obesos, respectivamente $(74,3 \pm 7,7 \mathrm{mmHg}$ vs. $65,4 \pm 9,4 \mathrm{mmHg}, \mathrm{p}=0,0002)$. Considerando a aferição da PAS 5', não foram encontradas diferenças entre adolescentes com e sem histórico de HAS materna e também entre adolescentes com e sem histórico de HAS paterna, respectivamente $(112,7 \pm 13,6 \mathrm{mmHg}$ vs. $113,8 \pm 13,0 \mathrm{mmHg}, \mathrm{p}=0,78 \mathrm{e}$ $115,6 \pm 10,9 \mathrm{mmHg}$ vs. $112,7 \pm 13,9 \mathrm{mmHg}, \mathrm{p}=0,30$ ). Da mesma forma, não foram encontradas diferenças na medida da PAD 5' entre adolescentes com e sem histórico de HAS materna e também entre adolescentes com e sem histórico de HAS paterna, respectivamente $(71,2 \pm 8,8 \mathrm{mmHg}$ vs. $70,0 \pm 9,8 \mathrm{mmHg}, \mathrm{p}=$ 0,61 e $73,5 \pm 10,25 \mathrm{mmHg}$ vs. $69,0 \pm 9,0 \mathrm{mmHg}, \mathrm{p}=$ $0,12)$. Encontramos diferenças entre as medidas da PAS basal e após 5 minutos de repouso, respectivamente $(125,3 \pm 12,1 \mathrm{mmHg}$ vs. $120,2 \pm 12,1 \mathrm{mmHg}, \mathrm{p}=$ 0,0000); e entre PAD basal e após 5 minutos de repouso, respectivamente $(78,7 \pm 7,5 \mathrm{mmHg}$ vs. $74,3 \pm 7,7 \mathrm{mmHg}, \mathrm{p}=0,0001$ ).

Vinte e cinco adolescentes [ 24 obesos $(63,2 \%)$ e 1 não obeso $(3,6 \%)$ ] apresentaram a alteração dermatológica conhecida como acanthosis nigricans. A média do IMC foi superior nos adolescentes que apresentaram acanthosis se comparado aos que não tiveram acanthosis, respectivamente $\left(34,7 \pm 7,2 \mathrm{Kg} / \mathrm{m}^{2}\right.$ vs. $\left.23,9 \pm 8,4 \mathrm{Kg} / \mathrm{m}^{2}, \mathrm{p}<0,01\right)$. A média da circunferência abdominal e da RCQ foram maiores no grupo com acanthosis do que no grupo sem acanthosis, respectivamente $(100,2 \pm 17,9 \mathrm{~cm}$ vs. $76,2 \pm 15,7 \mathrm{~cm}, \mathrm{p}=0,01$; $\mathrm{e}$ $0,88 \pm 0,09$ vs. e $0,77 \pm 0,06, p<0,01)$. Não ocorreram diferenças na glicemia basal entre o grupo de obesos e não obesos, respectivamente $(90,2 \pm 7,1 \mathrm{mg} / \mathrm{dl}$ vs. $90,8 \pm 9,9 \mathrm{mg} / \mathrm{dl}, \mathrm{p}=0,98)$. Não observamos diferença no colesterol do grupo de obesos se comparado ao grupo de não obesos, respectivamente (167,8 $\pm 34,2 \mathrm{mg} / \mathrm{dl}$ vs. $154,8 \pm 23,2 \mathrm{mg} / \mathrm{dl}, \mathrm{p}=0,10$ ). Os obesos apresentaram níveis de HDL inferiores aos não obesos, respectivamente $(36,5 \pm 10,5 \mathrm{mg} / \mathrm{dl}$ vs. $43,0 \pm 9,2 \mathrm{mg} / \mathrm{dl}, \mathrm{p}=0,0104)$. Os obesos apresentaram níveis superiores de triglicerídeos se comparados aos não obesos, respectivamente $(124,6 \pm 80,0 \mathrm{mg} / \mathrm{dl}$ vs. $74,2 \pm 31,4 \mathrm{mg} / \mathrm{dl}, \mathrm{p}=0,0016)$. A dosagem plasmática de ácido úrico foi superior no grupo de obesos se comparado aos não obesos, respectivamente $(5,8$ $\pm 1,4 \mathrm{mg} / \mathrm{dl}$ vs. $4,5 \pm 1,0 \mathrm{mg} / \mathrm{dl}, \mathrm{p}=0,0000$ ).

Quando analisamos o grupo de obesos em separado, encontramos um predomínio de adolescentes que chegaram ao serviço por vontade própria $(\mathrm{n}=15$, $39,5 \%)$. Outros $10(26,3 \%)$ foram encaminhados por profissional de saúde, enquanto que o mesmo número foi trazido por um familiar responsável. A questão da saúde foi apontada em mais da metade dos casos $(\mathrm{n}=$ $20,52,6 \%$ ) como motivo isolado para a procura do tratamento. Vinte e um pacientes $(55,3 \%)$ já haviam sido submetidos a algum tratamento anterior para perda de peso. Destes, a maioria $(n=19 ; 90,5 \%)$ através de medidas dietéticas (nutricionais) e em 14 $(73,4 \%)$ das vezes com acompanhamento de um profissional de saúde. Nove pacientes $(42,8 \%)$ dos que já haviam sido submetidos a tratamento prévio já tinham feito uso de medicamentos para este propósito. Na maioria dos casos $(n=7,77,8 \%)$ a medicação foi prescrita por um profissional de saúde.

Tabela 2. Dados antropométricos das populaçōes estudadas.

\begin{tabular}{llcc}
\hline Variável & $\begin{array}{c}\text { Obesos } \\
(\mathbf{n}=\mathbf{3 8})\end{array}$ & $\begin{array}{c}\text { Não obesos } \\
(\mathbf{n}=\mathbf{2 8})\end{array}$ & $\begin{array}{c}\text { Significância } \\
(\mathbf{p})\end{array}$ \\
\hline IMC (Kg/m $\mathbf{2})$ & $33,9 \pm 7,7$ & $19,6 \pm 2,4$ & - \\
Cintura (cm) & $98,9 \pm 15,8$ & $66,9 \pm 5,2$ & 0,0000 \\
RCQ & $0,86 \pm 0,08$ & $0,74 \pm 0,04$ & 0,0000 \\
Prega Triciptal (mm) & $38,2 \pm 9,2$ & $16,1 \pm 7,1$ & 0,0000 \\
Prega Subescapular (mm) & $34,4 \pm 9,8$ & $13,2 \pm 7,1$ & 0,0000 \\
Relação Sub/Tric & $0,92 \pm 0,18$ & $0,85 \pm 0,28$ & 0,15 \\
\hline
\end{tabular}

Valores expressos em média $\pm S D$; $I M C$, índice de massa corporal; Cintura, medida da circunferência abdominal; $R C Q$, relação cintura quadril; Rel. Sub/Tric, relação entre a prega subescapular e a prega triciptal. 
Tabela 3. Dados clínicos e laboratoriais das populaçōes estudadas.

\begin{tabular}{lccc}
\hline Variável & $\begin{array}{c}\text { Obesos } \\
(\mathbf{n}=\mathbf{3 8})\end{array}$ & $\begin{array}{c}\text { Não obesos } \\
(\mathbf{n}=\mathbf{2 8})\end{array}$ & $\begin{array}{c}\text { Significância } \\
(\mathbf{p})\end{array}$ \\
PAS basal $(\mathbf{m m H g})$ & $125,4 \pm 11,9$ & $112,6 \pm 8,5$ & 0,0000 \\
PAS 5' (mmHg) & $120,2 \pm 12,0$ & $105,1 \pm 8,9$ & 0,0000 \\
PAD basal (mmHg) & $78,8 \pm 7,4$ & $67,3 \pm 8,9$ & 0,0000 \\
PAD 5' (mmHg) & $74,4 \pm 7,7$ & $64,8 \pm 9,0$ & 0,0000 \\
Colesterol (mg/dl) & $167,8 \pm 34,4$ & $154,8 \pm 23,2$ & 0,10 \\
HDL (mg/dl) & $36,5 \pm 10,5$ & $43,0 \pm 9,2$ & 0,0104 \\
LDL (mg/dl) & $107,5 \pm 31,0$ & $99,7 \pm 21,3$ & 0,35 \\
Triglicerídeos (mg/dl) & $124,6 \pm 80,0$ & $74,2 \pm 31,4$ & 0,0024 \\
Ácido Úrico (mg/dl) & $5,8 \pm 1,4$ & $4,5 \pm 1,0$ & 0,0000 \\
\hline
\end{tabular}

Valores expressos em média+SD; PAS basal, Pressão arterial sistólica no tempo zero; PAS 5', Pressão arterial sistólica após 5 minutos de repouso; PAD basal, Pressão arterial diastólica no tempo zero; PAD 5; Pressão Arterial diastólica após 5 minutos de repouso.

\section{DISCUSSÃO}

O presente trabalho se caracteriza como um estudo em corte transverso de uma população de adolescentes obesos e não obesos, atendida em uma unidade especializada de um Hospital Universitário do Rio de Janeiro. Possivelmente, os dados obtidos não refletem a realidade da população do município. A adolescência é uma fase da vida na qual a classificação de obesidade se torna dificultosa, uma vez que a correlação entre índices antropométricos e o percentual e a distribuição de gordura corporal podem variar sensivelmente de acordo com a raça e o estágio de maturação sexual $(6,21)$. Para definição de obesidade, foi utilizado o trabalho que melhor reflete a realidade da população de adolescentes da região sudeste brasileira (18). A utilização do IMC se justifica por sua boa correlação com o percentual de gordura corporal, praticidade de execução e reprodutibilidade, o que pode ser visto em diversos trabalhos $(14,20,22)$. Nossa população de obesos teve, além obviamente do IMC, a medida da cintura e a RCQ superiores aos índices encontrados na população de não obesos. Isto possivelmente caracteriza uma distribuição truncal da gordura corporal na nossa amostra de obesos, fato já observado anteriormente (21).

Além da perspectiva de se tornar um adulto obeso, nossa amostra de adolescentes obesos apresentou alterações clínico-metabólicas em maior freqüência que o grupo de não obesos. A lesão dermatológica acanthosis nigricans predominou no grupo de obesos. Notou-se pois uma associação significativa entre acanthosis e IMC, medida da cintura e RCQ. É conhecida a relação entre obesidade e acanthosis nigricans $(15,19,23)$ e existem evidências de que na adolescência, obesos com acanthosis podem ter maior resistência à ação da insulina do que obesos sem esta lesão (15). As medidas da PAS 5' e da PAD 5' foram significativa- mente superiores no grupo dos adolescentes obesos. Encontramos valores superiores de PA quando ela foi aferida no tempo zero, isto é, no exato momento em que $o$ adolescente assumiu a posição supina. A aferição tanto da PAS como da PAD quando realizada após um período de repouso comumente registra níveis menores e isto já foi descrito na literatura $(24,25)$, e por isso adotamos em nossa metodologia, a mensuração da PA após repouso de 5 minutos. A obesidade associa-se indubitavelmente com a hipertensão arterial $(26,27)$. É considerada fator de risco para HAS (26), tanto que dentro das estratégias que objetivam controle da pressão arterial, a redução do peso é considerada medida terapêutica inicial (24). De fato, reduções ponderais mesmo modestas resultam em reduções nos níveis de PA em grande parte dos indivíduos hipertensos $(28,29)$, e podem ser consideradas um fator que otimiza a ação de drogas anti-hipertensivas (30).

Encontramos valores superiores de TG e ácido úrico nos obesos. É conhecida a associação da obesidade com a elevação dos níveis de TG $(7,31,32)$. Sabese também que esta associação pode ser encontrada antes da idade adulta $(11,12)$. Apesar de o TG não ser considerado um fator de risco independente para doença coronariana (33), seu excesso pode estar associado a redução nos níveis de $\mathrm{HDL}$, o que favorece o fenômeno de "enriquecimento" do LDL com TG. O LDL "enriquecido" de TG sofre ação da lipase hepática resultando em partículas menores e mais densas de LDL e com maior poder aterogênico (34). Existem evidências de que estas partículas estão associadas a um risco elevado para doença coronariana (35). Além disso, o estado de hipertrigliceridemia parece estar associado a distúrbios da atividade fibrinolítica, também relacionados com a incidência de doença coronariana (36). O excesso de peso também está relacionado à elevação dos níveis de ácido úrico e à ocor- 
rência de gota. Embora esses achados provenham de análises de populações adultas $(37,38)$, acreditamos que poderíamos evidenciar a ocorrência destes distúrbios após um longo prazo de acompanhamento da nossa amostra de adolescentes.

Encontramos valores de HDL menores no grupo de obesos. A associação entre excesso ponderal e niveis reduzidos de HDL é conhecida e já foi citada anteriormente $(31,32)$. Ocorre porém, que esta associação pode ser encontrada antes da idade adulta (39). Isto se torna importante uma vez que valor do HDL como fator protetor do desenvolvimento de doença coronariana é descrito em diversos estudos $(40,41)$; e parece ser mais pronunciado em indivíduos do sexo feminino (42).

Quando o grupo de obesos foi analisado em separado, observou-se nesses adolescentes uma preocupação com a questão da saúde. Boa parte deles buscaram o programa movidos pelo seu próprio interesse e, em mais da metade dos casos, a preocupação com a saúde foi o motivo isolado para querer perder peso, fato que contrasta com relatos publicados que celebram a questão da estética como grande motivador para perda de peso em adolescentes $(43,44)$. Uma boa parcela dos pacientes já tinha sido submetida a tratamentos anteriores para perder peso, e na maioria com auxílio de um profissional de saúde, o que pode reforçar a importância da preocupação com a saúde encontrada nesses indivíduos. $O$ desejo de emagrecer nesta fase da vida, sobretudo no sexo feminino, pode estar associado à origem de distúrbios do comportamento alimentar, sendo a bulimia e a anorexia nervosa os mais conhecidos $(2,43)$. As questões da saúde e da estética atuaram de forma conjunta como fatores de motivação para a busca da perda ponderal nos adolescentes estudados. Chamou-nos a atenção o fato de um número significativo de adolescentes já terem utilizado alguma medicação visando a perda ponderal e, na maioria das vezes, com a participação de um profissional de saúde. De fato a obesidade deve ser considerada uma doença crônica e de difícil condução terapêutica (44). A utilização de medicamentos para perder peso deve ser criteriosa. Hoje valorizam-se as medidas que envolvam alterações nos hábitos de vida, sobretudo antes da idade adulta (45).

Em resumo, os resultados obtidos a partir da análise da presença da obesidade em adolescentes nos permite afirmar que vários distúrbios clínico-metabólicos desfavoráveis, relacionados com o excesso ponderal em populações de adultos, podem ter início antes dessa fase da vida. A análise dos impactos que a obesidade pode causar em adolescentes nos deixa cada vez mais certos de que quanto mais precoce for a implementação da terapêutica mais benefícios poderão ser alcançados. Apesar de os trabalhos da literatura correlacionarem o desejo de emagrecer com questões estéticas $(43,44)$, o fato de termos encontrado um percentual significativo de adolescentes interessados na perda ponderal visando benefícios em sua saúde, ratifica a necessidade do controle ponderal nesta fase da vida.

\section{REFERÊNCIAS}

1. Kuczmarski RJ. Increasing prevalence of overweight among US adults: the National Health and Nutrition Examination Surveys, 1960 to 1991. JAMA 1992;272:205-15.

2. Williamson DF. Descriptive epidemiology of body weight and weight change in U.S, adults. Ann Int Med 1993: 119:646-9.

3. Prentice AM, Jebb SA. Obesity in Britain: Gluttony or sloth? BMJ 1995;311:437-9.

4. Sichieri R, Coitinho DC, Leão MM. High temporal, geographic, and income variation in body mass index among adults in Brazil. Am J Pub Health;84:793-8.

5. WHO Expert Committee on Physical Status: the use and interpretation of Anthropometry Physical status: the use and interpretation of anthropometry: Report of a WHO expert committee. (WHO technical report series; 854) World Health Organization 1995.

6. Travers SH. Gender and Tanner stage differences in body composition and insulin sensitivity in early pubertal children. J Clin Endocrinol Metab 1995; 172-8.

7. WHO. Obesity, Preventing and managing the global epidemic. Report of a WHO consultation on obesity. Genève, 3-5 jun 1997.

8. Abrahan S, Nordiesk M. Relationship of excess weight in children and adults. Public Health Rep 1960;75:263-73.

9. Charney E, Goodman HC, McBride M, Lyon B, Pratt R. Childhood antecedents of adult obesity. Do chubby infants become obese adults? N Engl J Med 1976;295:6-9.

10. Stunkard AJ et al. An adoption study of human obesity. N Engl J Med 1986;314.

11. Epstein LH, McCurley J, Wing RR, Valoski A. Five-year follow-up of family-based behavioral treatments for childhood obesity, J Consult Clin Psychol 1990;58:661-4.

12. Freedman DS et al. Relationship of changes in obesity to serum lipid and lipoprotein changes in childhood and adolescence. JAMA 1985:254:515-20.

13. Jiang $X$, Srinivasan $S$, Webber LS, Wattigney WA, Berenson OS. Association of fasting insulin level with serum lipid and lipoprotein level in children, adolescents, and young adults: The Bogalusa heart study. Arch Intern Med 1995: 155: 190-6.

14. Rosner B et al. Percentiles for body mass index in U.S children 5 to 17 years of age. J Pediatr 1998:132:211-22.

15. Ilyés I, Pósán E, Sári S. Insulin resistance in obese boys with acanthosis nigricans. Acta Paed Hung 1992;32:325-32. 
16. Report of the Second Task Force on Blood Pressure Control in Children. Pediatrics; 79:7-25.

17.Anjos LA. Índice de massa corporal (massa corporal $x$ estatura ${ }^{2}$ ) como indicador do estado nutricional de adultos: revisão de literatura. Rev Saúde Pública 1992;26:431-6.

18.Sichieri R, Allam VLC. Avaliação do estado nutricional de adolescentes brasileiros através do índice de massa corporal. J Ped 1996;72.

19.Stuart CA et al. Insulin resistance with acanthosis nigricans: the roles of obesity and androgen excess. Metabolism 1986;35:197-205.

20. Friedewald WT, Levy RI, Fredrickson DS. Estimation of low-density lipoprotein cholesterol in plasma without use of preparative ultracentrifuge. Clin Chem 1972;499-502.

21. Daniels SR et al. The utility of body mass index as a measure of body fatness in children and adolescents: differences by race and gender. Pediatrics 1997;99:804-7

22. Stamler $\mathrm{R}$ et al. Weight and blood pressure. Findings in hypertension screening of 1 million Americans. J Am Clin Assoc 1978:240.

23. Richards GE et al. Obesity, acanthosis nigricans, insulin resistance, and hyperandrogenemia: pediatric perspective and natural history. J Pediatr 1995;893-7

24. The Sixth Report of the Joint National Committee on Prevention, Detection, Evaluation, and Treatment of High Blood Pressure, NIH publication, nov. 1997.

25. Silva JR GR, Clemente EL, Gomes MB. Variabilidade da pressão arterial de consultório em pacientes com Diabetes Mellitus do tipo I. Arq Bras Endocrinol Metab 1999:43:96-103.

26. Hsueh WA, Buchanan TA. Obesity and hypertension Endocr Metab Clin North Am 1994;23:405-27.

27. Lucas CP, Estigarribia JA, Darga LL, Reaven GM. Insulin and blood pressure in obesity. Hypertension 1985;7:702-6.

28. Trials of Hypertension Prevention Collaborative Research Group. Effects of weight loss and sodium reduction intervention on blood pressure and hypertension incidence in overweight people with high normal blood pressure: the Trials of Hypertension Prevention, phase II. Arch Inter Med 1997; 157:657-67

29. Whelton PK et al. Efficacy of weight loss and reduced sodium intake in the trial of nonpharmacologic interventions in the elderly (TONE). Circulation :94:1-18.

30. Neaton JD et al. Treatment of mild hypertension study: final results. J Am Clin Assoc 1993:270:713-24.

31. Ochard TJ, Becker DJ, Bates M, Kuller LH, Drash AL, Plasma insulin and lipoprotein concentrations: an atherogenic association? Am J Epidemiol 1983;326-37.
32. Zavaronl I, Bonora $E$, Pagliara $M$ et al. Risk factors for coronary artery disease in healthy persons with hyperinsulinemia and normal glucose tolerance. $\mathbf{N}$ Engl J Med 1989:320:702-6.

33. Hulley SB et al. Epidemiology as a guide to clinical decisions. The association between triglyceride and coronary heart disease. N Engl J Med 1980;302:1383-9.

34. Sniderman AD. Association of hyperapobetalipoproteinemia with endogenous hypertriglyceridemia and atherosclerosis. Ann Intern Med 1892;833-9.

35. Austin MA et al. Low-density lipoprotein subclass patterns and risk of myocardial infarction. JAMA 1988:260:1917-21.

36. Hamsten A. Increased plasma levels of a rapid inhibito of tissue plasminogen activator in young survivors of myocardial infarction. N Engl J Med 1985;313:1557-63.

37. Davis MA et al. Body fat distribution and osteoarthritis. Am J Epidemiol 1990;701-7.

38. Roubennoff R. Incidence and risk factors for gout in white men. JAMA 266:3004-7.

39. Burns TL. Increased familial cardiovascular mortality in obese school children: the Muscatine Ponderosity Family Study. Pediatrics 1992;89:262-8.

40. NIH Consensus Conference: Triglyceride, High-density Lipoprotein and Coronary Heart Disease. JAMA 1993:269:505-10.

41. Stampfer MJ. A prospective study of cholesterol, apolipoproteins and the risk of myocardial infarction. $\mathbf{N}$ Engl J Med 1991;325:373-81

42. Kannel WB. Metabolic risk factors for coronary heart disease in women: perspective from the Framingham Study. Am Heart J 1987: 1 14:413-9.

43. Moses N, Banilivy MM, Lifshitz F. Fear of obesity among adolescent girls. Pediatrics 1989;83:393-8.

44. Serdula MK et al. Weight control practices of U.S. adolescents and adults. Ann Intern Med 1993; 1 19:667-71.

45. Hoerr SML, Nelson RA, Essex-Sorlie D. Treatment and follow-up of obesity in adolescent girls. J Adolesc Health 1988;9:28-37.

\section{Endereço para correspondência:}

João R.I. Carneiro

Rua Eng. Alfredo Modrach 261

22221-130 Rio de Janeiro, RJ 